\title{
Magnetic resonance sentinel lymph node imaging and magnetometer-guided intraoperative detection in prostate cancer using superparamagnetic iron oxide nanoparticles
}

This article was published in the following Dove Press journal:

International Journal of Nanomedicine

\author{
Alexander Winter \\ Tobias Kowald ${ }^{2}$ \\ Tina Susanne Paulo \\ Philipp Goos' \\ Svenja Engels' \\ Holger Gerullis' \\ Jonas Schiffmann' \\ Ajay Chavan ${ }^{2}$ \\ Friedhelm Wawroschek' \\ 'University Hospital for Urology, \\ Klinikum Oldenburg, School of \\ Medicine and Health Sciences, Carl \\ von Ossietzky University Oldenburg, \\ Oldenburg, Germany; ${ }^{2}$ Institute \\ of Diagnostic and Interventional \\ Radiology, Klinikum Oldenburg, \\ Oldenburg, Germany
}

Purpose: Sentinel lymph node (LN) dissection (sLND) using a magnetometer and superparamagnetic iron oxide nanoparticles (SPION) as a tracer was successfully applied in prostate cancer (PCa). The feasibility of sentinel LN (SLN) visualization on MRI after intraprostatic SPION injection has been reported. In the present study, results of preoperative MRI identification of SLNs and the outcome of subsequent intraoperative magnetometer-guided sLND following intraprostatic SPION injection were studied in intermediate- and high-risk PCa.

Patients and methods: A total of 50 intermediate- and high-risk PCa patients (prostatespecific antigen $>10 \mathrm{ng} / \mathrm{mL}$ and/or Gleason score $\geq 7$ ) scheduled for radical prostatectomy with magnetometer-guided sLND and extended pelvic LND (eLND), were included. Patients underwent MRI before and one day after intraprostatic SPION injection using T1-, T2-, and T2*-weighted sequences. Diagnostic rate per patient was established. Distribution of SLNs per anatomic region was registered. Diagnostic accuracy of sLND was assessed by using eLND as a reference standard.

Results: SPION-MRI identified a total of 890 SLNs (median 17.5; IQR 12-22.5). SLNs could be successfully detected using MRI in all patients (diagnostic rate 100\%). Anatomic SLN distribution: external iliac $19.2 \%$, common iliac $16.6 \%$, fossa obturatoria $15.8 \%$, internal iliac $13.8 \%$, presacral $12.1 \%$, perirectal $12.0 \%$, periprostatic $3.7 \%$, perivesical $2.3 \%$, and other regions $4.4 \%$. LN metastases were intraoperatively found in 15 of 50 patients $(30 \%)$. sLND had a $100 \%$ diagnostic rate, $85.7 \%$ sensitivity, $97.2 \%$ specificity, $92.3 \%$ positive predictive value, $94.9 \%$ negative predictive value, false negative rate $14.3 \%$, and $2.8 \%$ additional diagnostic value (LN metastases only outside the eLND template).

Conclusion: MR scintigraphy after intraprostatic SPION injection provides a roadmap for intraoperative magnetometer-guided SLN detection and can be useful to characterize a reliable lymphadenectomy template. Draining LN from the prostate can be identified in an unexpectedly high number, especially outside the established eLND template. Further studies are required to analyze discordance between the number of pre- and intraoperatively identified SLNs.

Keywords: lymphoscintigraphy, magnetic resonance imaging, magnetometer, prostate cancer, sentinel lymphadenectomy, SPION

University Hospital for Urology, Klinikum

Oldenburg, School of Medicine and

Health Sciences, Carl von Ossietzky University Oldenburg, Rahel-Straus-Str.

10, 26133 Oldenburg, Germany

Tel +49 44I 4032302

Fax +49 44I 4032303

Email winter.alexander@klinikum-

oldenburg.de

\section{Introduction}

Pelvic lymph node (LN) dissection (LND) is still the gold standard for LN staging in clinically localized prostate cancer (PCa). The prevalence of LN involvement is directly related to the number of dissected LNs or extent of the LND. ${ }^{1}$ However, the 
rate of complications rises along with the number of LNs removed. $^{2}$

Because of therapeutic consequences and the increased complication rate of the extended LND (eLND) as well as the low detection rate of limited LND procedures, Wawroschek et al transferred techniques and concepts of targeted radioisotope-guided sentinel LN (SLN) identification in other tumor entities to $\mathrm{PCa}{ }^{3}$ As established for breast cancer, conventional use in $\mathrm{PCa}$ patients involves radioactive marking of SLNs with ${ }^{99 m}$ Technetium nanocolloid, lymphoscintigraphy for preoperative evaluation, and a gamma probe for intraoperative SLN detection. ${ }^{4}$ Planar lymphoscintigraphy, or single-photon emission CT (SPECT), provides the surgeon with important preoperative information on location and number of SLNs. ${ }^{5}$ A high sensitivity for identification of LN metastases could be demonstrated for this radioisotopeguided sentinel procedure in $\mathrm{PCa}{ }^{6-9}$

Nevertheless, the pros of the current SLN detection procedure also come with some serious cons. The dependence on radioisotopes limits the application of this procedure to small parts of the developed world, and it imposes restrictions on patient planning and hospital logistics. The procedure exposes patients, as well as surgical staff, to ionizing radiation emitted by the technetium-based tracer material. The spatial resolution of lymphoscintigraphy is quite limited ( 7-8 mm), which makes identification of smaller LNs, typical of pelvic LNs, difficult. Furthermore, the differentiation of SLNs, especially in the periprostatic, presacral, and perirectal region is difficult because of high periprostatic activity and excreted radiotracer in the bladder.

To overcome these issues, superparamagnetic iron oxide nanoparticles (SPION) were tested and applied successfully for intraoperative detection of SLNs in breast cancer. ${ }^{10}$ Combining magnetic tracer with other novel sentinel techniques (eg, indocyanine green fluorescence) is under investigation. ${ }^{11} \mathrm{We}$ presented the first results on intraoperative detection of SLNs using intraprostatic SPION injection and a handheld magnetometer in patients with $\mathrm{PCa} .{ }^{12}$ However, to be able to perform an entirely radioisotope-free sentinel procedure in PCa patients, an alternative for conventional lymphoscintigraphy is needed.

SPION causes a loss of signal intensity ("negative contrast") on T2*(susceptibility)-weighted MRI sequences. ${ }^{13}$ Animal and human studies demonstrated that MRI after interstitial injection of SPION can be used as an alternative approach to lymphoscintigraphy for preoperative SLN evaluation. ${ }^{14-17}$ In animal studies, the feasibility of MRI SLN imaging with gadofosveset trisodium-albumin and ferumoxytol as an intraprostatic contrast agent was shown. ${ }^{18,19}$
A Phase I study of ferumoxytol for MR lymphography in PCa patients was carried out. ${ }^{20}$ In order to develop a magnetic alternative for preoperative localization of SLNs, a preliminary study determined if intraprostatic SPION injection can identify SLNs draining the prostate on MRI in PCa patients. ${ }^{21}$

After the safety and feasibility of SLN visualization using MRI in PCa has been shown, the present study assesses the results of preoperative MR SLN imaging and the diagnostic accuracy of magnetometer-guided sentinel LND (sLND) in intermediate- and high-risk PCa patients. A pathological correlation was performed based on eLND as a reference standard.

\section{Patients and methods Study design}

A total of 50 consecutive patients with intermediate- or high-risk PCa (prostate-specific antigen [PSA] $>10 \mathrm{ng} / \mathrm{mL}$ and/or Gleason score $\geq 7$ ) scheduled to undergo transrectal SPION injection before magnetometer-guided sLND and radical retropubic prostatectomy at our university center were recruited for this study conducted between March 2015 and January 2016. Patients with known intolerance or hypersensitivity to iron or dextran compounds, with iron overload disease, or with pacemakers or other implantable devices in the chest wall were excluded.

\section{Magnetic SPION tracer}

The used Sienna $+{ }^{\circledR}$ tracer is a component of the SentiMag ${ }^{\circledR}$ system (Endomagnetics Ltd, Cambridge, UK). This system for marking and identifying SLNs comprises a handheld magnetometer, the SentiMag ${ }^{\circledR}$ unit itself, and the Sienna+ ${ }^{\circledR}$ magnet tracer. Sienna $+{ }^{\circledR}$ is classified as a class IIa medical device by Medical Device Directive 93/42/EEC. Each milliliter of Sienna ${ }^{\circledR}$ contains circa 28 milligrams of iron. The particles have a carboxydextran coating and a mean hydrodynamic diameter of $60 \mathrm{~nm}$. Sienna $+{ }^{\circledR}$ has comparable functional properties to ${ }^{99 \mathrm{~m}}$ Technetium nanocolloid. Like the radionuclide, the tracer flows through the lymph system and gets trapped in the SLNs after interstitial injection.

Regarding non-clinical toxicology, Sienna $+{ }^{\circledR}$ has been reviewed and tested as specified in EN 10993-1:2009 based on the specified site of injection and duration. Sienna $+{ }^{\circledR}$ is contraindicated in any patient with hypersensitivity to iron oxide or dextran compounds and should not be administered in any patient with an iron overload disease or with a metal implant close to the expected SLN location. Sienna $+{ }^{\circledR}$ is only approved for interstitial injection. When similar material to that used in Sienna $+{ }^{\circledR}$ has been injected intravenously, the 
following undesirable effects have been reported: Common $(<2 \%)$ - pain at the injection site, vasodilation, and paresthesia. Uncommon ( $\geq 0.1 \%$ to $<1 \%$ ) - asthenia, back pain, injection site reactions, chest pain, nausea, vomiting, headache, taste perversion, pruritus, and rash. Rare ( $\geq 0.01 \%$ to $<0.1 \%$ ) - hypersensitivity and anaphylaxis, hypertension, phlebitis, hyperesthesia, anxiety, dizziness, convulsion, parosmia, dyspnea, increased cough, rhinitis, eczema, and urticaria. There have been a small number of reports of inflammatory and hypersensitivity response with intradermal injection. There is no evidence of adverse reaction following interstitial injection. ${ }^{22}$

\section{Tracer injection}

The injection technique in PCa differs from that in other tumor entities. In breast cancer and malignant melanoma, a peritumoral injection is performed. In $\mathrm{PCa}$, which often occurs multifocally, it is difficult to clearly define the index lesion and the part of the organ that the metastatic spread originates from. Hence, prostate lymphoscintigraphy aimed to visualize all LNs draining the prostate, under which the SLN of cancer also exist.

One day prior to surgery, a total of $2 \mathrm{~mL}$ of SPION $\left(\right.$ Sienna $\left.+{ }^{\circledR}\right)$ were injected into prostate using transrectal ultrasound guidance. The tracer was evenly distributed as three deposits each on the left and the right side of the prostate as described previously. ${ }^{12}$

\section{SPION-MRI}

Pelvic MRI was performed before and a day after intraprostatic SPION injection. All studies were conducted using a 1.5-T MRI Scanner (MAGNETOM Aera; Siemens, Erlangen, Germany). Transversal T1-weighted spin echo, T2-weighted turbo spin echo, T2-weighted fat-sat turbo spin echo, and diffusion-weighted images of the pelvis were obtained. In addition, Short-T1 inversion recovery sequences in the coronal plane and pre- and post-contrast $\mathrm{T} 2 *$-weighted gradient echo scans in the transversal plane were carried out to localize the LNs with SPION uptake. The MRI parameters are shown in Table 1.

The pre- and post-contrast T2*-weighted gradient echo images were compared to identify the LNs showing loss of signal intensity after SPION uptake. The pre-SPION MRI was considered as negative control and to exclude artifacts, which could have been misinterpreted as LNs (eg, blood vessels). Any LN with a drop in signal intensity due to SPION uptake was considered as a SLN. The MRI images were analyzed separately by two experienced radiologists using Centricity PACS Workstation (GE Healthcare, Little Chalfont, UK). Inter-observer disagreement was resolved by consensus.

\section{Surgical procedure and histopathological examination}

At surgery, magnetometer (SentiMag $\left.{ }^{\circledR}\right)$-guided sLND and eLND were followed by radical retropubic prostatectomy. All cases were performed by two different high-volume surgeons, who used the same anatomic template during eLND. The eLND template included the area along the external iliac vessels up to the femoral canal distally and up to the bifurcation of the common iliac artery proximally. In addition, all lymphatic fatty tissue around the common iliac artery bifurcation and along the internal iliac artery, within the obturator fossa and from the area dorsal of the obturator nerve was removed, as described by Weingärtner et al. ${ }^{23}$ The lateral limit consisted of the pelvic sidewall, and the medial dissection limit was defined by perivesical fat.

Table I Parameters of the SPION-MRI sequences obtained before and after intraprostatic SPION injection for identification of sentinel lymph nodes in prostate cancer patients

\begin{tabular}{|c|c|c|c|c|c|c|}
\hline \multirow[t]{2}{*}{ Parameters } & \multicolumn{6}{|l|}{ Sequences } \\
\hline & STIR & TIw SE & T2w TSE & T2w fat-sat TSE & T2* GRE (hemo) & DW-EPI \\
\hline Plane & Coronal & Transversal & Transversal & Transversal & Transversal & Transversal \\
\hline TR/TE & $5,350 / 67 \mathrm{~ms}$ & $659 / 10 \mathrm{~ms}$ & $3,500 / 89 \mathrm{~ms}$ & $3,500 / 89 \mathrm{~ms}$ & $\begin{array}{l}40 / 1.26 \mathrm{~ms} \\
40 / 2.86 \mathrm{~ms}\end{array}$ & $2,734 / 61 \mathrm{~ms}$ \\
\hline Flip angle & $150^{\circ}$ & $141^{\circ}$ & $160^{\circ}$ & $160^{\circ}$ & $20^{\circ}$ & N/A \\
\hline Slice thickness & $4 \mathrm{~mm}$ & $4 \mathrm{~mm}$ & $4 \mathrm{~mm}$ & $4 \mathrm{~mm}$ & $4 \mathrm{~mm}$ & $4 \mathrm{~mm}$ \\
\hline FOV & $380 \mathrm{~mm}$ & $400 \mathrm{~mm}$ & $400 \mathrm{~mm}$ & $400 \mathrm{~mm}$ & $400 \mathrm{~mm}$ & $400 \mathrm{~mm}$ \\
\hline Matrix & 256 & 256 & 320 & 320 & 208 & 128 \\
\hline Imaging time & $2: 31$ minutes & 2:48 minutes & 2:58 minutes & 2:40 minutes & $3: 14$ minutes & $\mathrm{I}: 56$ minutes \\
\hline
\end{tabular}

Abbreviations: DW, diffusion weighted; EPI, echo planar imaging; FOV, field of view; GRE, gradient-recalled echo; SE, spin echo; SPION, superparamagnetic iron oxide nanoparticles; STIR, short-tau inversion recovery; TE, echo time; TR, repetition time; TSE, turbo; N/A, not applicable. 
During sLND, all metal retractors were removed from the surgical field and polymer retractors (SUSI ${ }^{\circledR}$, Aesculap ${ }^{\circledR}$; B. Braun Melsungen AG, Melsungen, Germany) were used, in order to avoid any interference with the SentiMag ${ }^{\circledR}$ probe. All SLNs detected by the SentiMag ${ }^{\circledR}$ magnetometer were removed; each magnetically active $\mathrm{LN}$ was seen as a SLN. LNs other than SLNs directly adjoining and adhering to SLNs were also removed, if an in-situ separation was not possible. Thereafter, an eLND was conducted to remove the remaining lymphatic fatty tissue from the previously named regions.

Postoperatively, all resected LNs (SLNs and non-SLNs) were initially cut in $3 \mathrm{~mm}$ transverse sections, routinely processed, and completely embedded in paraffin, while 4-5 $\mu \mathrm{m}$ thick sections were stained with $\mathrm{H} \& \mathrm{E}$.

\section{Outcome measures}

\section{MR SLN imaging}

A positive procedure was defined as a detection of at least one SLN by MRI after intraprostatic injection of SPION. This served to determine the detection rate (patients with at least one detected SLN/total number examined). Number and localization of the SLNs were documented.

\section{Magnetometer-guided sLND}

Outcomes for diagnostic test accuracy were diagnostic rate, sensitivity, specificity, positive predictive value (PPV), negative predictive value (NPV), and false positive and false negative (FN) rates, all measured at patient level. False negative cases were defined as patients with histologically negative SLN while cancer was found in other LNs. False positive cases were defined as patients with SLNs containing metastases outside the eLND template while the eLND template did not reveal any metastases. ${ }^{9}$ Thus, false positive rate provides a measure of the additional diagnostic value of sLND over and aforementioned eLND. In addition, the proportion of histologically positive cases in SLND only was determined.

A $2 \times 2$ table with sLND as the index test and eLND as the reference standard was used to calculate sensitivity, specificity, NPV, and PPV.

\section{Ethical approval}

All subjects gave their written informed consent for inclusion before they participated in the study. The study was conducted in accordance with the Declaration of Helsinki, and the protocol was registered in an international clinical trials register (UIN: research registry 2857). The study was approved by the Medical Ethics Committee of the Carl von Ossietzky University Oldenburg (no 2017-006).

\section{Results}

The study recruited 50 intermediate- or high-risk PCa patients scheduled to undergo intraprostatic SPION injection before magnetometer-guided SLND, eLND, and radical prostatectomy. Table 2 summarizes the patient characteristics. The total PSA was $9.84 \mathrm{ng} / \mathrm{mL}$ (median; IQR 5.89-14.53). LN metastases were found in 15 of the 50 patients (30\%), with a median of two positive LNs (IQR 1-2).

None of the 50 patients exhibited adverse events attributable to the Sienna $+{ }^{\circledR}$ injection. As seen in exemplary conducted histopathological verifications, LNs with SPION uptake (Figure 1) showed a strong drop of signal intensity on post-contrast $\mathrm{T}^{*}$-weighted images (Figure 2). SLNs could be successfully detected by SPION-MRI in all patients (50/50) resulting in a detection rate of $100 \%$. Tracer deposits in the prostate did not affect LN imaging in any patient. SPION injection identified a total of 890 SLNs. The median number of detected SLNs was 17.5 (IQR 12-22.5). An unexpected high number of SLNs was localized, especially in the presacral (12.13\%) and the perirectal (12.02\%) region. Figures 3 and 4 show the distribution of all SLNs per anatomic region in detail.

Considering the eLND template as a reference standard, results of sLND were $100 \%$ for diagnostic rate, $85.7 \%$ for sensitivity, $97.2 \%$ for specificity, $92.3 \%$ for PPV, $94.9 \%$ for NPV, and $14.3 \%$ for FN rate. Two of the $15 \mathrm{LN}$-positive patients showed histologically negative SLN while cancer was found in other LNs. On the other hand, in one of the $15 \mathrm{LN}$-positive patients, SLND showed an additional diagnostic value. In this case, sLND detected one LN metastasis outside the extended template while the eLND did not reveal any metastases (false positive rate $2.8 \%$ ). The percentage of $\mathrm{LN}$-positive patients with only metastases in SLN(s) was $80 \%(n=12)$.

\section{Discussion}

The feasibility of intraoperative detection of SLNs using intraprostatic SPION injection and a magnetometer system could be demonstrated in $\mathrm{PCa} .{ }^{12}$ Preoperative evaluation of SLNs in PCa patients based on this new magnetic approach as an alternative to lymphoscintigraphy was still pending. However, preoperative SLN identification offers the surgeon a roadmap with solid information on individual location of draining LNs. Recently, the safety and feasibility of preoperative evaluation of SLNs in PCa patients using intraprostatic 
Table 2 Patient characteristics

\begin{tabular}{|c|c|c|c|}
\hline Characteristics & $\begin{array}{l}\text { Overall } \\
n=50\end{array}$ & $\begin{array}{l}\text { Patients with negative } \\
\text { LNs } n=35(70 \%)\end{array}$ & $\begin{array}{l}\text { Patients with positive } \\
\text { LNs n }=15(30 \%)\end{array}$ \\
\hline $\begin{array}{l}\text { Age, years (median) } \\
\text { IQR }\end{array}$ & $\begin{array}{l}66.5 \\
61-72\end{array}$ & $\begin{array}{l}66 \\
60-71\end{array}$ & $\begin{array}{l}72 \\
64-74\end{array}$ \\
\hline $\begin{array}{l}\text { Total PSA, ng/mL (median) } \\
\text { IQR }\end{array}$ & $\begin{array}{l}9.84 \\
5.89-14.53\end{array}$ & $\begin{array}{l}9.61 \\
5.09-12.54\end{array}$ & $\begin{array}{l}10.27 \\
7.18-24.34\end{array}$ \\
\hline $\begin{array}{l}\text { No of LN removed (median) } \\
\text { IQR }\end{array}$ & $\begin{array}{l}16.5 \\
|3-2|\end{array}$ & $\begin{array}{l}16 \\
|2-2|\end{array}$ & $\begin{array}{l}\mid 8 \\
|5-2|\end{array}$ \\
\hline $\begin{array}{l}\text { No of SLN removed (median) } \\
\text { IQR }\end{array}$ & $\begin{array}{l}9 \\
6-12\end{array}$ & $\begin{array}{l}8 \\
5-12\end{array}$ & $\begin{array}{l}11 \\
7-13\end{array}$ \\
\hline $\begin{array}{l}\text { No of positive LN (median) } \\
\text { IQR }\end{array}$ & - & - & $\begin{array}{l}2 \\
1-2\end{array}$ \\
\hline \multicolumn{4}{|l|}{ Tumor stage (\%) } \\
\hline $\begin{array}{l}\text { T1c } \\
\text { T2a } \\
\text { T2b } \\
\text { T2c } \\
\text { T3 }\end{array}$ & $\begin{array}{l}28(56) \\
2(4) \\
6(12) \\
13(26) \\
1(2)\end{array}$ & $\begin{array}{l}24(68.57) \\
\text { I }(2.86) \\
4(11.43) \\
5(14.29) \\
\text { I }(2.86)\end{array}$ & $\begin{array}{l}4(26.67) \\
1(6.67) \\
2(13.33) \\
8(53.33) \\
0(0)\end{array}$ \\
\hline \multicolumn{4}{|l|}{ Biopsy Gleason score (\%) } \\
\hline $\begin{array}{l}6(3+3) \\
7(3+4) \\
7(4+3) \\
\geq 8\end{array}$ & $\begin{array}{l}9(18) \\
24(48) \\
9(18) \\
8(16)\end{array}$ & $\begin{array}{l}9(25.7 I) \\
19(54.29) \\
7(20) \\
0(0)\end{array}$ & $\begin{array}{l}0(0) \\
5(33.33) \\
2(13.33) \\
8(53.33)\end{array}$ \\
\hline \multicolumn{4}{|l|}{ Postoperative Gleason score (\%) } \\
\hline $\begin{array}{l}6(3+3) \\
7(3+4) \\
7(4+3) \\
\geq 8\end{array}$ & $\begin{array}{l}\text { I (2) } \\
26(52) \\
13(26) \\
10(20)\end{array}$ & $\begin{array}{l}\text { I (2.86) } \\
24(68.57) \\
8(22.86) \\
2(5.71)\end{array}$ & $\begin{array}{l}0(0) \\
2(13.33) \\
5(33.33) \\
8(53.33)\end{array}$ \\
\hline \multicolumn{4}{|l|}{ Pathologic stage (\%) } \\
\hline $\begin{array}{l}\text { pT2 } \\
\text { PT3a } \\
\text { pT3b } \\
\text { pT4 }\end{array}$ & $\begin{array}{l}27(54) \\
12(24) \\
10(20) \\
1(2)\end{array}$ & $\begin{array}{l}26(74.29) \\
6(17.14) \\
2(5.71) \\
1(2.86)\end{array}$ & $\begin{array}{l}\text { I }(6.67) \\
6(40) \\
8(53.33) \\
0(0)\end{array}$ \\
\hline
\end{tabular}

Abbreviations: LN, lymph node; PSA, prostate specific antigen.

SPION injection as an alternative to lymphoscintigraphy has been reported. ${ }^{21}$ The present study was able to show, that this approach is suited for MRI visualization of LNs draining the prostate in intermediate- and high-risk PCa patients scheduled for magnetometer-guided sLND. Therefore, SPION-MRI provides in combination with intraoperative use of a handheld magnetometer an entirely radiation-free technique for SLN identification in PCa.

SPION injection could preoperatively identify SLNs in all patients. By using the same SPION-MRI approach, Pouw et al successfully identified SLNs in 10/11 patients with breast cancer. ${ }^{17}$ For lymphoscintigraphy after intraprostatic injection of ${ }^{99 \mathrm{~m}}$ Technetium nanocolloid, Holl et al showed a detection rate of $97.6 \%{ }^{7}$ Besides the high detection reliability of the radiation-free SPION approach in our study, SPION injection is safe, simple, and can be performed alone by a urologist. The required MRI sequences go hand in hand with a comparable low effort, too.

The number of SLNs visualized by SPION-MRI in our study was higher than described in previous studies using radioisotope-tracer and lymphoscintigraphy or SPECT. 6,7,24,25 Several possible causes are imaginable. Lymphoscintigraphy has a limited spatial resolution. In contrast, the high spatial resolution of MRI allows individual differentiation of SLNs adjacent to each other, which appear as one hotspot in lymphoscintigraphy. MRI is highly sensitive to very small concentrations of SPION and very small SLNs could be visualized. Maybe, the smaller size of SPION (60 nm; ${ }^{99 m}$ Technetium nanocolloid: $<80 \mathrm{~nm}$ ) could result in marking of secondary landing sites, too. 

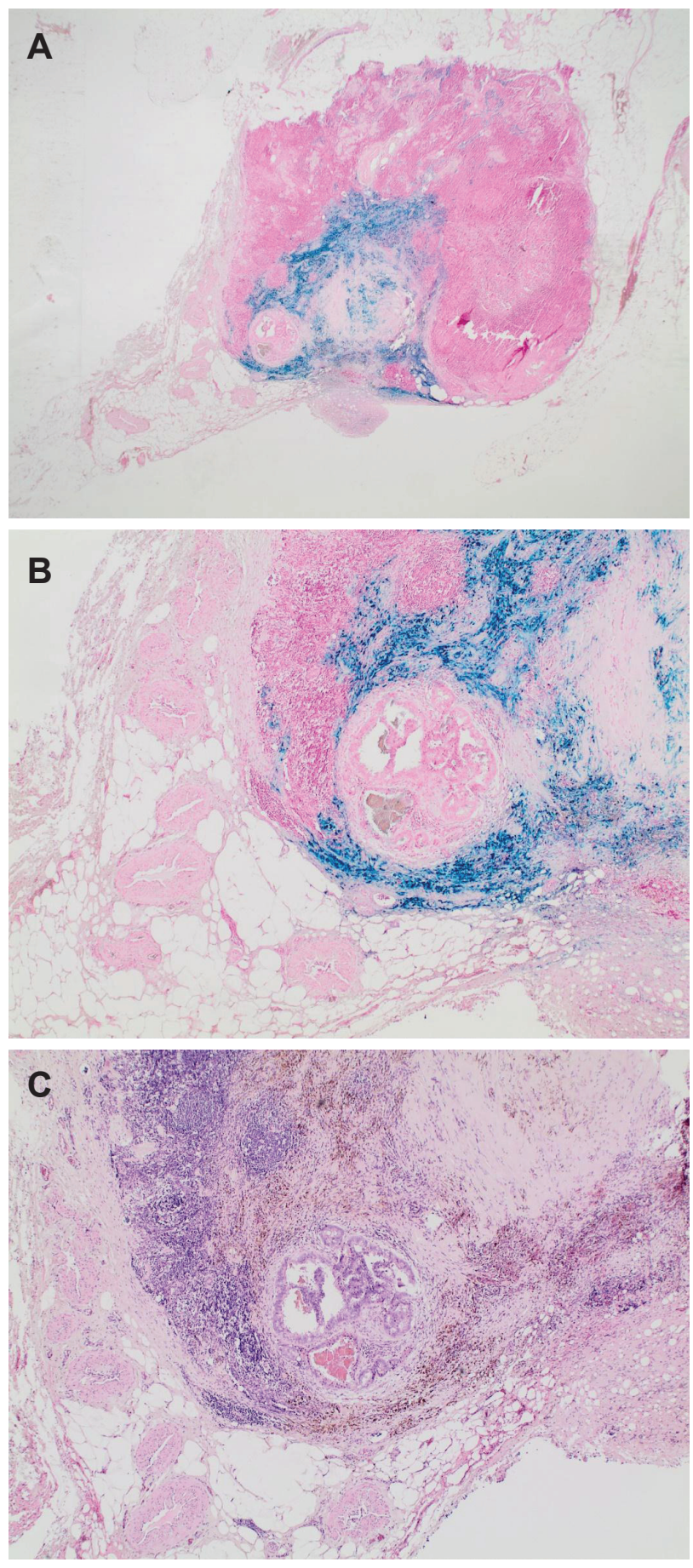

Figure I Examples of a sentinel lymph node, which showed a strong drop of signal intensity on T2*-weighted MRI after intraprostatic SPION injection. Perl's Prussian blue (A, B) and H\&E (C) staining shows blue and brown discoloration of the absorbed iron oxide nanoparticles in the lymphatic tissue. In the center of the image, a 4-mm metastasis of a Gleason 8 prostate cancer.

Notes: Magnification A, I.25x; B, 4x; C, 4x.

Abbreviation: SPION, superparamagnetic iron oxide nanoparticles.

In the present study, magnetometer-guided sLND identified a lower number of SLNs as visualized preoperatively by MRI. In breast cancer, preoperative MRI after interstitial SPION injection showed the same number of marked SLNs as found during surgery in 6/11 patients (55\%). Two patients

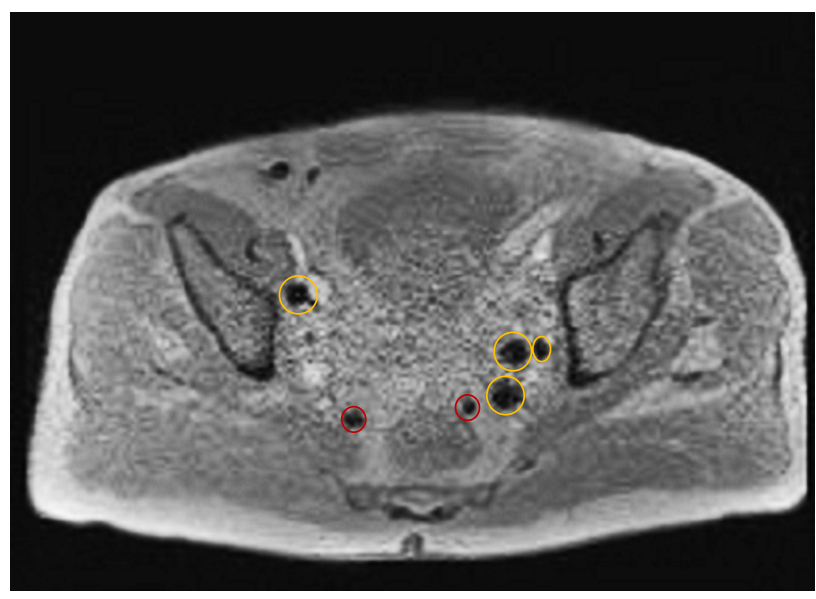

Figure 2 After intraprostatic administration of SPION, multiple SLNs, including the two red marked perirectal nodes showed a strong drop of signal intensity on postcontrast $\mathrm{T} 2 *$-weighted image.

Abbreviations: SLN, sentinel lymph node; SPION, superparamagnetic iron oxide nanoparticles.

showed one additional SLN on MRI that was not identified during surgery. ${ }^{17}$ In other breast cancer studies, the concordance rates between the number of SLNs identified by imaging (lymphoscintigraphy or SPECT) and during surgery ranged from $39 \%$ to $73 \%{ }^{26,27}$ Conventional lymphoscintigraphy tends to be associated with an underestimation of the SLN number while SPION-MRI overestimates the number of SLNs.

The decay of the magnetic signal is largely dependent on the size/shape of the signal sources or accumulation in the SLNs. The magnetometer probe is most effective when measuring nodes with tracer uptake concentrated close to the node surface where it can be measured at very close range. ${ }^{28}$ However, in the present study, the SLNs showed a very heterogeneous SPION uptake. In vivo, adipose tissue surrounding SLNs can limit the distance of the probe to the node resulting in insufficient exposure of the node or not sufficient measurement of the magnetic signal. Furthermore, the presence of tissue in the vicinity of the probe tip introduces a reduction in the magnetic signal in vivo due to the negative susceptibility of surrounding tissue. ${ }^{28}$ These effects and the fact that MRI is more sensitive to very small concentrations of SPION than the magnetometer may result in the higher number of SLNs visualized. Some of the positive nodes visualized using MRI could be secondary landing sites with only very low iron content resulting in a negative intraoperative measurement. In animal studies, negatively measured secondary Prussian blue stained nodes could be seen to contain only a small level of magnetic tracer. ${ }^{28}$ In a porcine model, the mean iron content of the SLNs detected using the same magnetometer was determined to be 265 (SD 206) $\mu \mathrm{g} .{ }^{29}$ 


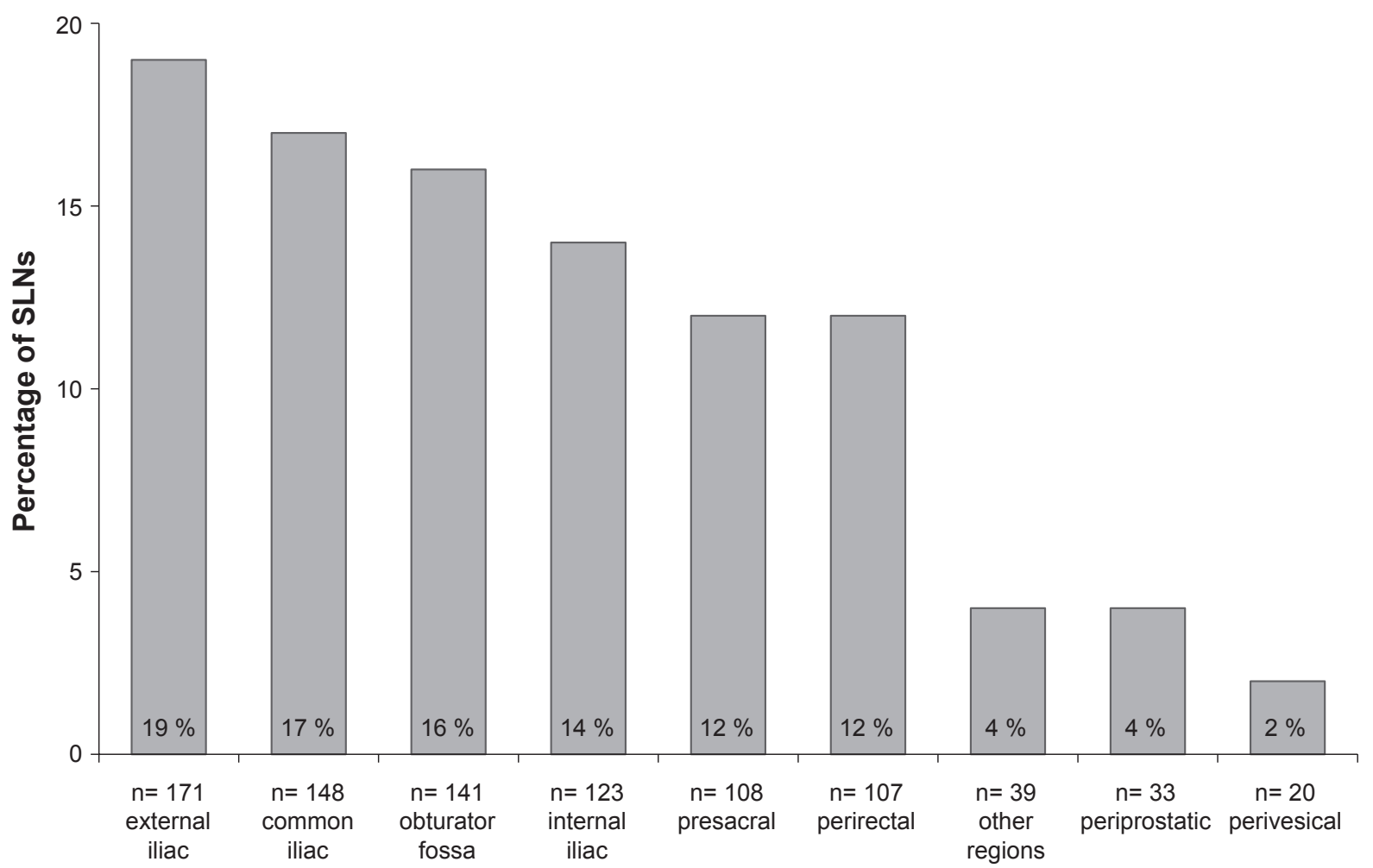

Figure 3 Anatomical distribution of 890 sentinel lymph nodes of the prostate of 50 patients with intermediate- or high-risk prostate cancer based on MRI after intraprostatic SPION injection.

Abbreviations: SLN, sentinel lymph node; SPION, superparamagnetic iron oxide nanoparticles.

For a novel magnetometer probe, designed using advanced magnetic tunneling junction sensing technology at a fixed distance of $4.0 \mathrm{~mm}$ from the signal source, a $50 \mu \mathrm{g}$ limit of detection was calculated. ${ }^{28}$ Unfortunately, a quantification of the amount of magnetic tracer or content of iron in the detected SLNs (eg, vibrating sample magnetometry ${ }^{29}$ ) for the clarification of threshold values in PCa patients is still pending.

In addition, the limited spatial resolution of the SentiMag ${ }^{\circledR}$ probe $(\sim 20 \mathrm{~mm})$ could restrain the differentiation of SLN signals from the injection site's background. The higher resolution of novel probes using magnetic tunneling junction techniques (resolution $\sim 4 \mathrm{~mm}$ ) could lead to an improvement in intraoperative SLN detection. ${ }^{28}$

By using magnetic marking and MRI in the present study, a very high proportion of SLNs could be visualized outside the established eLND template. A total of $24 \%$ of SPION marked nodes were found one-half each in the presacral and perirectal region. A significant number of SLNs could be visualized outside the standard node template in studies dealing with the radioactive marking approach, too. However, in most of these studies, the number of SLNs located in the perirectal and presacral region was much lower. ${ }^{25,30}$ On the other hand, Joniau et al have shown that $21 \%$ of preoperatively detected SLNs could be found in these two regions (presacral: 7\%; perirectal: 14\%). In the same study, $8 \%$ of LN-positive patients would have been missed if a lymphadenectomy in the presacal region had not been carried out. ${ }^{24}$

A current systematic literature review has shown that sLND has a diagnostic accuracy compared with eLND. ${ }^{9}$ This is also underlined by the results of a recent head-tohead comparison of three LN invasion predicting eLND- or sLND-based nomograms. ${ }^{31}$ In the present study, two of 15 LN-positive patients could not be detected by magnetometerguided sLND. One of the two false-negative patients had a high-volume Gleason 9 cancer, in accordance with previous reports showing poorer outcomes of sLND in highly aggressive tumors. ${ }^{32}$ Accordingly, a lower median sensitivity and a higher $\mathrm{FN}$ rate were observed in other studies, including only intermediate- and high-risk $\mathrm{PCa} .{ }^{9}$

On the other hand, the present and other studies show an increasing detection of positive nodes, when combining eLND with sLND or individualized extension of LND outside the borders of eLND. Therefore, in high-risk disease, sLND should be combined with eLND. ${ }^{33}$

The main limitation of the present study is that a direct LN-related comparison between SPION-MRI results and histopathological outcome is still pending. Therefore, the 


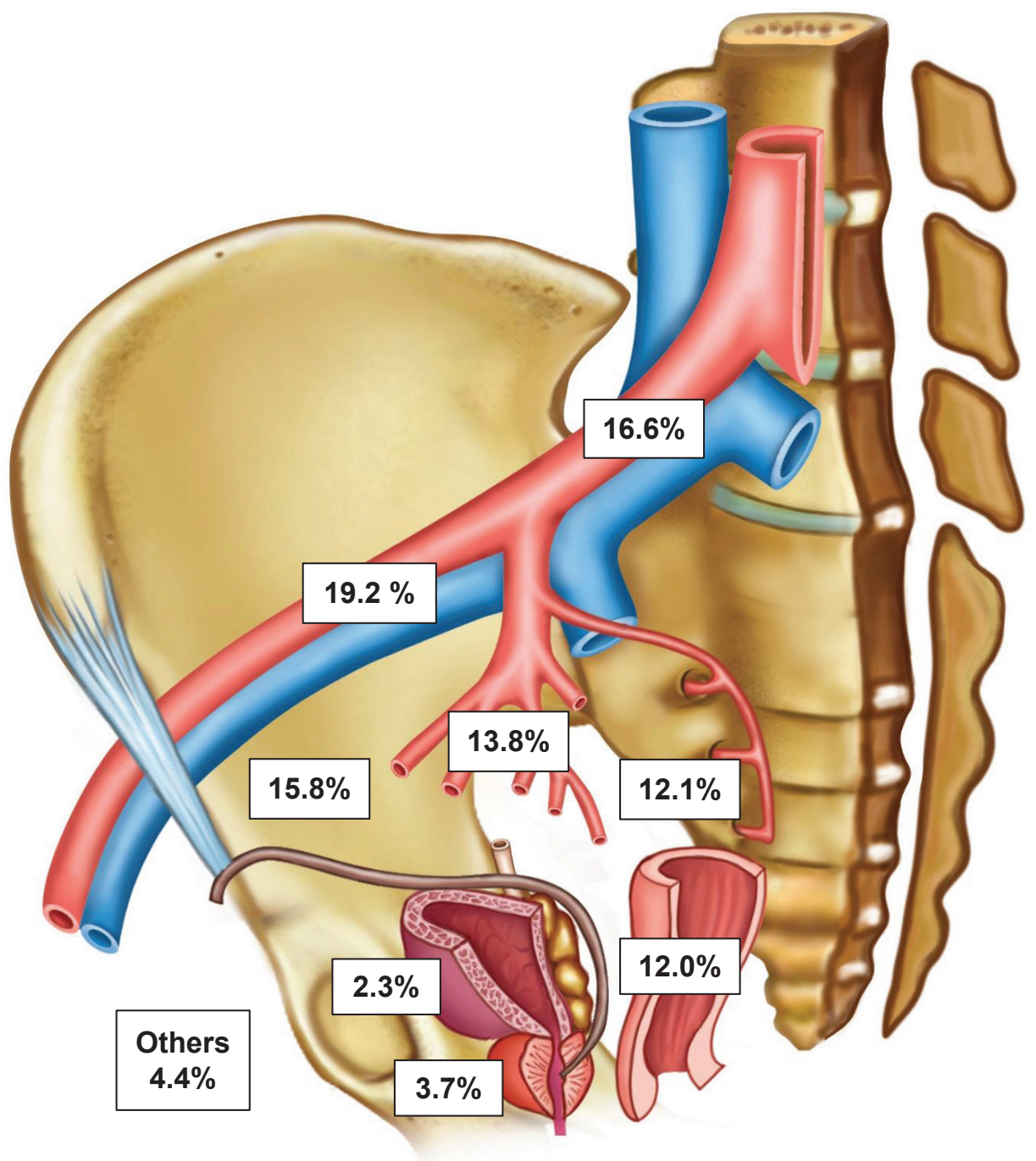

Figure 4 Anatomical distribution and localization of 890 sentinel lymph nodes of the prostate of 50 patients with intermediate- or high-risk prostate cancer based on MRI after intraprostatic SPION injection.

Abbreviation: SPION, superparamagnetic iron oxide nanoparticles.

potential of MRI for non-invasive identification of LN metastases after intraprostatic SPION administration has not been investigated. MRI after intravenous administration of ultrasmall superparamagnetic iron oxide (USPIO) has been successfully used as a non-invasive method for evaluating metastatic LN involvement in PCa. ${ }^{34,35}$ Intravenously injected USPIOs are taken up in healthy LN tissue and not in metastatic areas, and consequently, areas affected by tumors have relatively high signal intensity on $\mathrm{T} 2$-weighted images compared with normal tissue. With respect to intraprostatic injection of SPION, the results of Motomura et al are promising in this regard. ${ }^{36}$ After interstitial SPION injection $\left(\right.$ Resovist $\left.^{\circledR}\right)$ in patients with clinically node-negative breast cancer $(\mathrm{n}=70)$, all 19 patients with a finding of metastasis in SLNs at pathology were also shown to have metastases on MRI. Forty-eight of 50 patients with non-metastatic
SLNs diagnosed at pathology were classified as having nonmetastatic nodes on MRI. On a patient-by-patient basis, the sensitivity, specificity, and accuracy of MRI for the diagnosis of SLN metastases were $100 \%, 96 \%$, and $97 \%$, respectively. However, in the present study, histopathological examinations of SLNs after intraprostatic SPION injection showed a very heterogeneous SPION uptake regardless of metastasis also in healthy LN tissue (Figure 1). Therefore, in PCa, MRI after intraprostatic SPION injection appears to be suitable for the visualization of SLNs, but unsuitable for the direct detection of metastases in SLNs. For this issue, further systematic analysis is required.

The FN rate of the sentinel procedure in PCa was shown to correlate with Gleason scores. ${ }^{7}$ One fundamental problem of the SLN approach is that when LNs are fully metastasized or when the lymph pathways are blocked, the afferent lymph 
will be redirected to other LNs/non-SLNs. ${ }^{37}$ Patients with high-risk disease undergoing radio-guided SLND could thus have a lower number of SLNs or FN SLNs. ${ }^{32}$ That is why we are planning an analysis which correlate the number/ site of SLNs with different risk groups in PCa patients who underwent MR SLN imaging and magnetic sLND.

A sample size calculation was not performed. However, based on previous studies, the included collective $(n=50)$ could be expected to have a sufficient number of SLNs to visualize and analyze $(\sim 350 \mathrm{SLNs}) .{ }^{12}$

\section{Conclusion}

sLND using a magnetometer and SPION as a tracer instead of radioisotopes was successfully applied in $\mathrm{PCa}$. In the present study, preoperative SLN visualization on MRI after intraprostatic SPION injection in intermediate- and high-risk PCa patients was shown. In combination with a handheld magnetometer system, SPION-MRI provides an entirely radiation-free technique for pre- and intraoparative SLN localization and has the potential to characterize a reliable lymphadenectomy template in PCa. The identification of an unexpectedly high number of SLNs, especially outside the extended node template and additional diagnostic value of sLND over and above eLND speak for the magnetic sentinel approach or the individualization of LND in intermediateand high-risk PCa. More work is needed to answer open questions - for example, through direct comparison of imaging results with LN-related histological outcome and detailed node-related analysis regarding the discordance between the number of pre- and intraoperatively identified SLNs.

\section{Acknowledgments}

Prospective studies on magnetometer-guided sLND were funded by the Research Pool of the Carl von Ossietzky University Oldenburg.

\section{Disclosure}

The author reports no conflicts of interest in this work.

\section{References}

1. Heidenreich A, Ohlmann $\mathrm{CH}$, Polyakov S. Anatomical extent of pelvic lymphadenectomy in patients undergoing radical prostatectomy. Eur Urol. 2007;52(1):29-37.

2. Briganti A, Chun FK, Salonia A, et al. Complications and other surgical outcomes associated with extended pelvic lymphadenectomy in men with localized prostate cancer. Eur Urol. 2006;50(5):1006-1013.

3. Wawroschek F, Vogt H, Weckermann D, Wagner T, Harzmann R. The sentinel lymph node concept in prostate cancer - first results of gamma probe-guided sentinel lymph node identification. Eur Urol. 1999; 36(6):595-600
4. Giammarile F, Alazraki N, Aarsvold JN, et al. The EANM and SNMMI practice guideline for lymphoscintigraphy and sentinel node localization in breast cancer. Eur J Nucl Med Mol Imaging. 2013;40(12): 1932-1947.

5. Goyal A, Mansel RE. Does imaging in sentinel node scintigraphic localization add value to the procedure in patients with breast cancer? Nucl Med Commun. 2005;26(10):845-847.

6. Winter A, Kneib T, Henke RP, Wawroschek F. Sentinel lymph node dissection in more than 1200 prostate cancer cases: rate and prediction of lymph node involvement depending on preoperative tumor characteristics. Int J Urol. 2014;21(1):58-63.

7. Holl G, Dorn R, Wengenmair H, Weckermann D, Sciuk J. Validation of sentinel lymph node dissection in prostate cancer: experience in more than 2,000 patients. Eur J Nucl Med Mol Imaging. 2009;36(9): 1377-1382.

8. Winter A, Kneib T, Wasylow C, et al. Updated nomogram incorporating percentage of positive cores to predict probability of lymph node invasion in prostate cancer patients undergoing sentinel lymph node dissection. J Cancer. 2017;8(14):2692-2698.

9. Wit EMK, Acar C, Grivas N, et al. Sentinel node procedure in prostate cancer: a systematic review to assess diagnostic accuracy. Eur Urol. 2017;71(4):596-605.

10. Karakatsanis A, Daskalakis K, Stålberg P, et al. Superparamagnetic iron oxide nanoparticles as the sole method for sentinel node biopsy detection in patients with breast cancer. Br J Surg. 2017;104(12):1675-1685.

11. Kuwahata A, Ahmed M, Saeki K, et al. Combined use of fluorescence with a magnetic tracer and dilution effect upon sentinel node localization in a murine model. Int J Nanomedicine. 2018;13:2427-2433.

12. Winter A, Woenkhaus J, Wawroschek F. A novel method for intraoperative sentinel lymph node detection in prostate cancer patients using superparamagnetic iron oxide nanoparticles and a handheld magnetometer: the initial clinical experience. Ann Surg Oncol. 2014; 21(13):4390-4396.

13. Stark DD, Weissleder R, Elizondo G, et al. Superparamagnetic iron oxide: clinical application as a contrast agent for MR imaging of the liver. Radiology. 1988;168(2):297-301.

14. Mizokami D, Kosuda S, Tomifuji M, et al. Superparamagnetic iron oxide-enhanced interstitial magnetic resonance lymphography to detect a sentinel lymph node in tongue cancer patients. Acta Otolaryngol. 2013;133(4):418-423.

15. Mccauley TR, Rifkin MD, Ledet CA. Pelvic lymph node visualization with MR imaging using local administration of ultra-small superparamagnetic iron oxide contrast. J Magn Reson Imaging. 2002;15(4): 492-497.

16. Iida S, Imai K, Matsuda S, et al. In vivo identification of sentinel lymph nodes using MRI and size-controlled and monodispersed magnetite nanoparticles. J Magn Reson Imaging. 2013;38(6):1346-1355.

17. Pouw JJ, Grootendorst MR, Bezooijen R, et al. Pre-operative sentinel lymph node localization in breast cancer with superparamagnetic iron oxide MRI: the SentiMAG Multicentre Trial imaging subprotocol. Br J Radiol. 2015;88(1056):2015063420150634. doi.

18. Turkbey B, Hoyt RF, Agarwal HK, et al. Magnetic resonance sentinel lymph node imaging of the prostate with gadofosveset trisodiumalbumin: preliminary results in a canine model. Acad Radiol. 2015 22(5):646-652.

19. Sankineni S, Smedley J, Bernardo M, et al. Ferumoxytol as an intraprostatic MR contrast agent for lymph node mapping of the prostate: a feasibility study in non-human primates. Acta Radiol. 2016;57(11):1396-1401.

20. Turkbey B, Agarwal HK, Shih J, et al. A Phase I Dosing Study of Ferumoxytol for MR Lymphography at $3 \mathrm{~T}$ in Patients With Prostate Cancer. AJR Am J Roentgenol. 2015;205(1):64-69.

21. Winter A, Chavan A, Wawroschek F. Magnetic resonance imaging of sentinel lymph nodes using intraprostatic injection of superparamagnetic iron oxide nanoparticles in prostate cancer patients: first-in-human results. Eur Urol. 2018;73(5):813-814.

22. Endomagnetics. Sienna $+^{\circledR}-$ Instruction for use. SIE-006, Issue 18, Multi-Language, 05/10/15. 
23. Weingärtner K, Ramaswamy A, Bittinger A, Gerharz EW, Vöge D, Riedmiller H. Anatomical basis for pelvic lymphadenectomy in prostate cancer: results of an autopsy study and implications for the clinic. J Urol. 1996;156(6):1969-1971.

24. Joniau S, van den Bergh L, Lerut E, et al. Mapping of pelvic lymph node metastases in prostate cancer. Eur Urol. 2013;63(3):450-458.

25. Mattei A, Fuechsel FG, Bhatta Dhar N, et al. The template of the primary lymphatic landing sites of the prostate should be revisited: results of a multimodality mapping study. Eur Urol. 2008;53(1):118-125.

26. Mathew MA, Saha AK, Saleem T, Saddozai N, Hutchinson IF, Nejim A. Pre-operative lymphoscintigraphy before sentinel lymph node biopsy for breast cancer. Breast. 2010;19(1):28-32.

27. Volders JH, van La Parra RF, Bavelaar-Croon CD, et al. Discordance between number of scintigraphic and perioperatively identified sentinel lymph nodes and axillary tumour recurrence. Breast. 2014;23(2): 159-164.

28. Cousins A, Balalis GL, Thompson SK, et al. Novel handheld magnetometer probe based on magnetic tunnelling junction sensors for intraoperative sentinel lymph node identification. Sci Rep. 2015;5:10842.

29. Pouw JJ, Ahmed M, Anninga B, et al. Comparison of three magnetic nanoparticle tracers for sentinel lymph node biopsy in an in vivo porcine model. Int J Nanomedicine. 2015;10:1235-1243.

30. Wawroschek F, Wagner T, Hamm M, et al. The influence of serial sections, immunohistochemistry, and extension of pelvic lymph node dissection on the lymph node status in clinically localized prostate cancer. Eur Urol. 2003;43(2):132-137.
31. Grivas N, Wit E, Tillier C, et al. Validation and head-to-head comparison of three nomograms predicting probability of lymph node invasion of prostate cancer in patients undergoing extended and/or sentinel lymph node dissection. Eur J Nucl Med Mol Imaging. 2017;44(13): 2213-2226.

32. Weckermann D, Dorn R, Holl G, Wagner T, Harzmann R. Limitations of radioguided surgery in high-risk prostate cancer. Eur Urol. 2007; 51(6):1549-1558.

33. van der Poel HG, Wit EM, Acar C, et al. Sentinel Node Prostate Cancer Consensus Panel Group members. Sentinel node biopsy for prostate cancer: report from a consensus panel meeting. BJU Int. 2017; 120:204-211.

34. Heesakkers RA, Hövels AM, Jager GJ, et al. MRI with a lymph-nodespecific contrast agent as an alternative to CT scan and lymph-node dissection in patients with prostate cancer: a prospective multicohort study. Lancet Oncol. 2008;9(9):850-856.

35. Birkhäuser FD, Studer UE, Froehlich JM, et al. Combined ultrasmall superparamagnetic particles of iron oxide-enhanced and diffusionweighted magnetic resonance imaging facilitates detection of metastases in normal-sized pelvic lymph nodes of patients with bladder and prostate cancer. Eur Urol. 2013;64(6):953-960.

36. Motomura K, Izumi T, Tateishi S, et al. Superparamagnetic iron oxideenhanced MRI at $3 \mathrm{~T}$ for accurate axillary staging in breast cancer. Br J Surg. 2016;103(1):60-69.

37. Morgan-Parkes JH. Metastases: mechanisms, pathways, and cascades. AJR Am J Roentgenol. 1995;164(5):1075-1082.
International Journal of Nanomedicine

\section{Publish your work in this journal}

The International Journal of Nanomedicine is an international, peerreviewed journal focusing on the application of nanotechnology in diagnostics, therapeutics, and drug delivery systems throughout the biomedical field. This journal is indexed on PubMed Central, MedLine, CAS, SciSearch ${ }^{\circledR}$, Current Contents ${ }^{\circledR} /$ Clinical Medicine,

\section{Dovepress}

Journal Citation Reports/Science Edition, EMBase, Scopus and the Elsevier Bibliographic databases. The manuscript management system is completely online and includes a very quick and fair peer-review system, which is all easy to use. Visit http://www.dovepress.com/ testimonials.php to read real quotes from published authors. 